\title{
Transgenic cassava resistance to African cassava mosaic virus is enhanced by viral DNA-A bidirectional promoter-derived siRNAs
}

\author{
Journal Article \\ Author(s): \\ Vanderschuren, Hervé; Akbergenov, Rashid; Pooggin, Mikhail M.; Hohn, Thomas; Gruissem, Wilhelm; Zhang, Peng \\ Publication date: \\ 2007 \\ Permanent link: \\ https://doi.org/10.3929/ethz-b-000007859
}

Rights / license:

In Copyright - Non-Commercial Use Permitted

Originally published in:

Plant Molecular Biology 64(5), https://doi.org/10.1007/s11103-007-9175-6 


\title{
Transgenic cassava resistance to African cassava mosaic virus is enhanced by viral DNA-A bidirectional promoter-derived siRNAs
}

\author{
Hervé Vanderschuren • Rashid Akbergenov • \\ Mikhail M. Pooggin • Thomas Hohn • \\ Wilhelm Gruissem • Peng Zhang
}

Received: 13 December 2006/Accepted: 16 April 2007/Published online: 10 May 2007

(C) Springer Science+Business Media B.V. 2007

\begin{abstract}
Expression of double-stranded RNA (dsRNA) homologous to virus sequences can effectively interfere with RNA virus infection in plant cells by triggering RNA silencing. Here we applied this approach against a DNA virus, African cassava mosaic virus (ACMV), in its natural host cassava. Transgenic cassava plants were developed to express small interfering RNAs (siRNA) from a CaMV 35S promoter-controlled, intron-containing dsRNA cognate to the common region-containing bidirectional promoter of ACMV DNA-A. In two of three independent transgenic lines, accelerated plant recovery from ACMV$\mathrm{NO} g$ infection was observed, which correlates with the presence of transgene-derived siRNAs 21-24 nt in length. Overall, cassava mosaic disease symptoms were dramatically attenuated in these two lines and less viral DNA accumulation was detected in their leaves than in those of wild-type plants. In a transient replication assay using leaf disks from the two transgenic lines, strongly reduced accumulation of viral single-stranded DNA was observed. Our study suggests that a natural RNA silencing mechanism targeting DNA viruses through production of virus-
\end{abstract}

H. Vanderschuren · W. Gruissem $\cdot$ P. Zhang $(\bowtie)$

Institute of Plant Sciences, ETH Zurich, Universitätstrasse 2,

Zürich 8092, Switzerland

e-mail: pzhang@ethz.ch;

R. Akbergenov · M. M. Pooggin · T. Hohn

Institute of Botany, University of Basel, Schönbeinstrasse 6,

Basel 4056, Switzerland

\section{P. Zhang}

Institute of Plant Physiology and Ecology, Shanghai Institutes

for Biological Sciences, Chinese Academy of Sciences, 300

Fenglin Road, Shanghai 200032, China

e-mail: zhangpeng@sibs.ac.cn derived siRNAs is turned on earlier and more efficiently in transgenic plants expressing dsRNA cognate to the viral promoter and common region.

Keywords African cassava mosaic virus - Cassava . RNA interference · Common region - Transcriptional gene silence $\cdot$ siRNA $\cdot$ Plant recovery

$\begin{array}{ll}\text { Abbreviations } \\ \text { ACMV } & \text { African cassava mosaic virus } \\ \text { AV1 } & \text { coat protein } \\ \text { BC1 } & \text { movement protein } \\ \text { CMD } & \text { cassava mosaic disease } \\ \text { CR } & \text { common region } \\ \text { dsDNA } & \text { double-stranded DNA } \\ \text { dsRNA } & \text { double-stranded RNA } \\ \text { Pro } & \text { bidirectional promoter of ACMV } \\ \text { PTGS } & \text { post-transcriptional gene silencing } \\ \text { RdRP } & \text { RNA-dependent RNA polymerase } \\ \text { REn/AC3 } & \text { replication enhancer protein } \\ \text { Rep/AC1 } & \text { replication associated protein } \\ \text { siRNA } & \text { small interfering RNAs } \\ \text { ssDNA } & \text { single-stranded DNA } \\ \text { TrAP/AC2 } & \text { transcriptional activator protein } \\ \text { TGS } & \text { transcriptional gene silencing } \\ \text { MYMV } & \text { Mungbean yellow mosaic virus } \\ \text { Wt } & \text { wild type }\end{array}$

\section{Introduction}

RNA interference provides a powerful tool to render plant resistant against virus infection via post-transcriptional 
and/or transcriptional gene silencing (PTGS and TGS; Waterhouse and Fusaro 2006). Expression of hairpin double-stranded RNA (dsRNA) homologous to coding sequences of RNA and DNA viruses has been shown to restrict viral infection in plants (Chen et al. 2004; Pandolfini et al. 2003; Wang et al. 2000). It is assumed that long dsRNA is processed by dicer proteins into small interfering RNAs (siRNA), which then target viral RNA for cleavage and degradation in a sequence-specific manner (Kalantidis et al. 2002; Smith et al. 2000). siRNAs have also been implicated in TGS, when Mette et al. (2000) found that dsRNA expression could trigger the methylation of a cognate target promoter sequence. This ability has been correlated with reduced transcription levels (Fojtova et al. 2003). Recently, we also proved that Mungbean yellow mosaic virus (MYMV)-infected Vigna mungo (black gram) plants were able to recover from the infection after bombardment with a construct expressing dsRNA targeting a viral sequence including the bidirectional promoter and the common region (CR, Pooggin et al. 2003). Since in geminiviruses the promoter region is normally not transcribed, the resistance might be caused by targeting the viral DNA. This provides a novel method to engineer DNA virus resistance in plants without targeting the coding sequence.

In order to investigate the efficacy of this approach in a stably transformed plant system, we produced transgenic cassava (Manihot esculenta Crantz) expressing hairpin dsRNA homologous to the sequences including the bidirectional promoter and CR of African cassava mosaic virus (ACMV), a begomovirus of family Geminiviridae. This virus, either alone or combined with other cassava geminiviruses, causes cassava mosaic disease (CMD), which has become the most important constraint to cassava production during the last decade in Africa (Legg and Thresh 2003). Because of its economical importance, breeding CMD resistance in cassava has been a key objective for cassava breeders. As an alternative to classical breeding programs, development of virus resistant cassava using transgenesis has been attempted in several biotechnology laboratories (Zhang et al. 2005; Chellappan et al. 2004a). Recently we demonstrated that transgenic cassava expressing anti-sense RNAs of ACMV Rep, TrAP and REn could resist ACMV infection via post-transcriptional gene silencing (Zhang et al. 2005). In this paper, we report the development of RNAi-based resistance to ACMV through expression of dsRNA homologous to its viral non-coding sequence. These results expand the potential of RNAi strategy against DNA viruses to their entire genome.

\section{Materials and methods}

Plasmid construction and cassava transformation

The binary expression vector pRNAi-dPro was constructed based on an RNAi plasmid described by Pooggin et al. (2003). The MYMV sequences were replaced with the ACMV-KE DNA-A sequence from position 21-277 (GenBank accession NC_001467; located between the transcription start sites, which includes an almost entire sequence of the common region with core elements of the leftward promoter and the adjacent 86 bp sequence with core elements of the rightward promoter) in the reverse and the forward orientations (Fig. 1a). The resulting construct was mobilized into Agrobacterium tumefaciens LBA4404 for transformation of cassava TMS60444 described by Zhang et al. (2000a).

\section{Characterization of transgenic lines}

Cassava genomic DNA was extracted from freeze-dried leaves according to Soni and Murray (1994). PCR and Southern analyses were carried out following standard protocols (Sambrook et al. 1989). Aliquots of $10 \mu \mathrm{g}$ genomic DNA were digested with or without $X b a \mathrm{I}$, which only cut the T-DNA once, for Southern analysis. The hybridization probe specific to CR of ACMV were DIGdUTP-labeled by PCR using a PCR DIG probe synthesis kit (Roche Diagnostics GmbH, Germany) according to the manufacturer's instructions. Primers used for PCR are listed in Table 1.

Transient viral replication assay

The infectious clones of ACMV-NOg DNA-A and DNA-B (Liu et al. 1997) were cloned into pbluescript (SK-) with two origins of replication in the tandem repeat sequences of the virus, respectively. Particle preparation and bombardment were conducted described by Zhang et al. (2000b). Equal amount of ACMV DNAA and DNA-B (200 ng/shot) were bombarded into leaf disks according to Zhang and Gruissem (2003). Around 100 leaf disks were used per treatment. Each experiment had 12 replicates. After post-cultivation, $6 \mu \mathrm{g}$ of total DNA from leaf disks were digested with DpnI to eliminate input DNA (dam-methylated), and SphI to linearize the DNA-A component. Southern blot was performed using a DIG-labelled probe specific to the ACMV-NOg AV1 gene. 


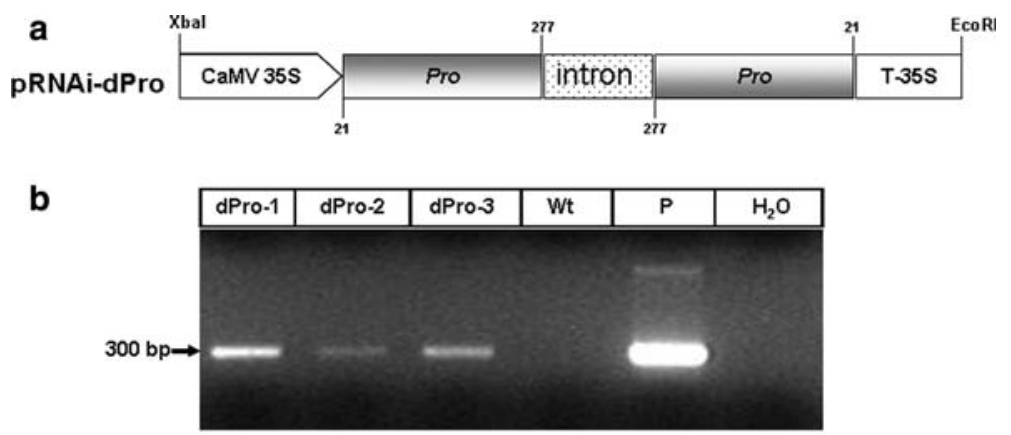

C

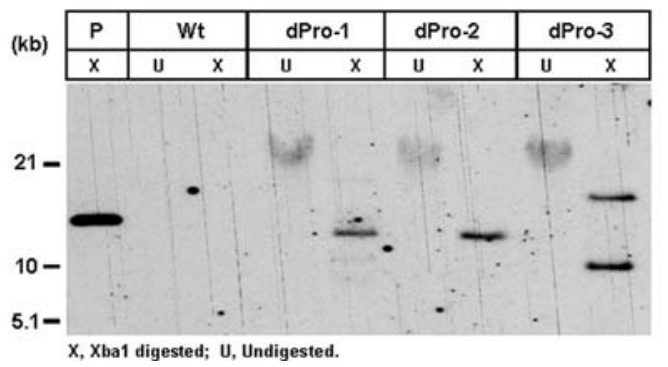

Fig. 1 Molecular analysis of cassava plants transformed with pRNAi-dPro. (a) The arrangement of hairpin double-stranded RNA expression cassette in construct pRNAi-dPro. The intergenic common region-containing promoter (Pro) sequences from position 21-277 of ACMV-KE (GenBank NC_001467) separated by a synthetic intron in the reverse and the forward orientations were inserted between CaMV $35 \mathrm{~S}$ promoter and terminator. Then the expression cassette was subcloned between $X b a \mathrm{I}$ and EcoRI of pCambia1300 to generate the
pRNAi-dPro. (b) Amplification of $300 \mathrm{bp}$ fragments between the CaMV 35S promoter and the intron of dsRNA expression cassette in transgenic cassava lines. (c) Transgene integration patterns detected in XbaI digested genomic DNA by corresponding DIG-labeled probes of ACMV DNA-A bidirectional promoter in transgenic cassava. P, control plasmid digested with $X b a \mathrm{I}$; Wt, wild-type control; Transgenic lines indicated by dPro- $x$

Table 1 The sequences and functions of primers and oligonucleotides used in the experiment

\begin{tabular}{|c|c|c|c|}
\hline & Name & Sequence & Function \\
\hline \multirow[t]{6}{*}{ Primers } & dPro- $5^{\prime}$ & 5'-GGACAGGGTACCAGCCACAACTTCGCCTGC-3' & \multirow[t]{2}{*}{ Transgene detection } \\
\hline & dPro-3' & 5'-AATCTTACCTCACGAGTGGTACAGCATCTCC-3' & \\
\hline & AV $1-5^{\prime}$ & 5'-CACCATGTCGAAGCGACCAGGAGATATCATC-3' & \multirow[t]{2}{*}{$A V 1$ probe amplification } \\
\hline & AV $1-3^{\prime}$ & 5'TTAATTGCCAATACTGTCATAGAAGTATATAC-3 & \\
\hline & $\mathrm{T} 7$ & 5'-TAATACGACTCACTATAG-3' & \multirow[t]{2}{*}{ Short RNA ladder preparation } \\
\hline & T7-NONA oligos & 5'-ACGGTTGGCCCCTTGGTTTCCCTATAGTGAGTCGTATTA-3' & \\
\hline \multirow[t]{4}{*}{ Oligonucleotides } & ACMV Pro sense & 5'-AGG GGC CAA CCG TAT AAT ATT ACC GGT-3' & \multirow[t]{2}{*}{ Promoter probes } \\
\hline & ACMV Pro antisense & 5'-ACC GGT AAT ATT ATA CGG TTG GCC CCT-3' & \\
\hline & ACMV AC1 sense & 5'-TACCTAAAGCACTTTAAAGAATTCATG-3' & $A C 1$ probe \\
\hline & ACMV BC1 sense & 5'-TGACACTGGGCCTAGATATAGGCCCAT-3' & $B C 1$ probe \\
\hline
\end{tabular}

\section{Plant infection with ACMV-NOg}

The 8-week-old transgenic and wild-type cassava plants developed from in vitro shoot cultures were used for ACMV infection. The plants usually had 8-12 leaves at that time. Before inoculation, the top expanded leaves are removed and only keep immature apical leaves. Infectious clones of ACMV-NOg were inoculated to apical meristems and immature leaves using a homemade particle inflow gun (PIG). Each plant was bombarded twice with $0.0625 \mathrm{mg}$ gold particles coated with $100 \mathrm{ng}$ of each ACMV component per bombardment as described (Zhang et al. 2005). After infection, cassava plants were kept at $28^{\circ} \mathrm{C}$ under $16 \mathrm{~h}$ light in the growth chamber.

Disease symptoms developing in inoculated and newly emerging leaves were visually recorded till the top leaves up to twelfth new leaf development. The symptom severity on fully expanded leaves was evaluated on a scale of $0-4$ described by Zhang et al. (2005). There was a minimum of 
4 plants per line in each infection test with three experimental repeats.

Virus detection in infected plants

Total DNA was extracted from the pooled 4th leaves of three plants 8 weeks after inoculation. Equal amounts of total DNA from each sample were digested with $S p h \mathrm{I}$ and analyzed using standard Southern blot protocol. Accumulated viral DNA was hybridized with a DIG-labelled probe specific to the ACMV-NOg AV1 gene. The intensity of bands was quantified by using AlphaImager ${ }^{\circledR}$ system (Alpha Innotech Corp).

\section{Small RNA detection}

Small RNA isolation and detection was performed as we described previously (Akbergenov et al. 2006). The DNA oligonucleotides used as the probes for siRNAs detection are listed in Table 1.

\section{Results}

Production of cassava transgenic lines

Transgenic cassava plants were regenerated from hygromycin-resistant somatic embryos developed from embryogenic cassava suspensions (Zhang et al. 2000a) that had been transformed with Agrobacterium tumefaciens LBA4404 harboring the binary vector pRNAi-dPro. The pRNAi-dPro contains a $256 \mathrm{bp}$ inverted repeat of the ACMV-KE DNA-A bidirectional promoter [containing the 169 bp common region (CR) shared with DNA-B], sense and anti-sense viral sequences being separated by a synthetic intron (Goodall and Filipowicz 1989) and driven by the strong CaMV $35 \mathrm{~S}$ promoter (Fig. 1a). The ACMV-KE sequence shares $96.1 \%$ identity with the corresponding region of ACMV-NOg. Even though more than 200 hygromycin-resistant somatic embryos were obtained from three independent transformation events, only three lines could be developed into plantlets.

The integration of the transgene T-DNA cassettes has been confirmed by PCR and Southern blot hybridisation in the three transgenic lines (Fig. 1b, c). Line dPro-3 had 2 integration events, while lines dPro- 1 and dPro- 2 had only one. The transgenic line dPro-1 showed reduced growth when compared to wild-type and other transgenic lines in agar, but not in soil. Similar phenomena of growth reduction have been observed in transgenic cassava expressing siRNAs to block expression of two cytochromes P450 CYP79D1 and CYP79D2 in the biosynthetic pathway for the cyanogenic glucosides (Jørgensen et al. 2005). The reason is unclear. In the greenhouse, all three transgenic plants developed a wild-type phenotype of above-ground part of the plants in pots. After harvesting the roots from older-than-6-month plants, all three lines showed normal rooting and storage root development in comparison with wild-type plants. For example, weights of storage roots from 9-month-old plants of wild-type, dPro-1 and dPro- 2 are $37.8 \pm 7.2,49.3 \pm 17.6$ and $26.1 \pm 6.1$ grams, respectively.

Impaired viral replication in leaf disk assays

In order to evaluate the virus resistance of these transgenic lines, we used a transient viral replication assay in cassava leaf disks. This method has been previously used for cassava to quantify the resistance level of different cassava cultivars and transgenic lines (Zhang and Gruissem 2003; Zhang et al. 2005). The results obtained showed strongly reduced accumulation (by $\geq 90 \%$ ) of ACMV-NOg DNA forms including double-stranded (ds) and single-stranded (ss) DNA molecules in the transgenic lines dPro-2 and dPro-3 as compared to wild-type cassava (Fig. 2). Interestingly, the relative reduction of ssDNA molecules was much more pronounced than that of dsDNA. There is no significant difference on the accumulation levels of viral dsDNA and ssDNA between dPro-2 and dPro-3 (Fig. 2, lanes 4 and 5).

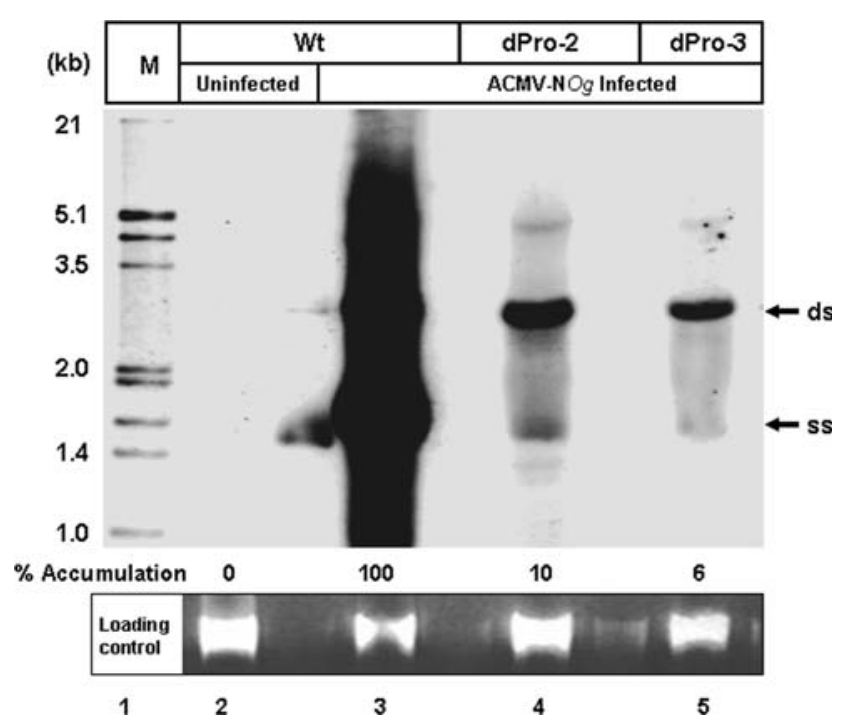

Fig. 2 Reduced viral DNA accumulation after biolistic inoculation of leaf discs with ACMV-NOg. Total DNA samples were digested with $S p h \mathrm{I}$ and $D p n I$. M, DIG-labeled molecular marker; Wt, wild-type control; Transgenic lines indicated by dPro-x. Viral DNA was detected using a DIG-labeled $A V 1$ probe. The positions of viral single-stranded (ss) and double-stranded (ds) DNA forms are indicated 
Transgenic cassava show enhanced recovery phenotype after ACMV infection

Transgenic and wild-type plants were inoculated with $200 \mathrm{ng}$ viral DNA of ACMV-NOg per plant under controlled growth chamber conditions. Symptom severity was determined according to our earlier standard scales (Zhang et al. 2005). ACMV-inoculated lines dPro-2 and dPro-3 displayed significant attenuation of symptom development and a reduction of symptom severity compared to the untransformed and dPro-1 transformed lines (Figs. 3, 4). Most of the emerging new leaves of lines dPro-2 and dPro3 showed a considerably reduced viral symptom severity and a fast phenotype recovery (Fig. 3a). The total symptom scores of dPro-2 and dPro-3 are significantly lower than that of wild-type (Fig. 3b). The line dPro-1 did not show resistance to the infection and displayed a similar profile as wild-type. The average levels of symptom severity in the first initial peak of wild-type, dPro- 1 and dPro-3 can reach 2.6, while for line dPro-2 the highest score is 1.6 (Fig. 3a). Both dPro-2 and dPro-3 plants showed similar symptom

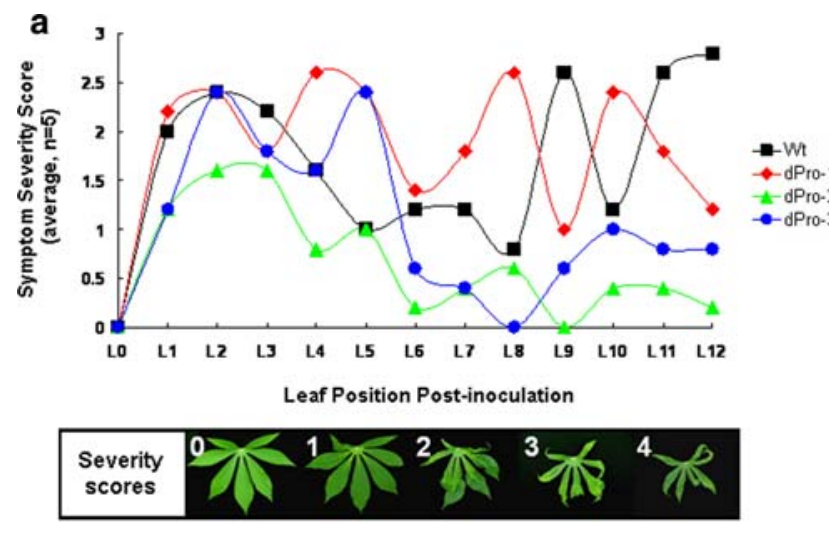

b

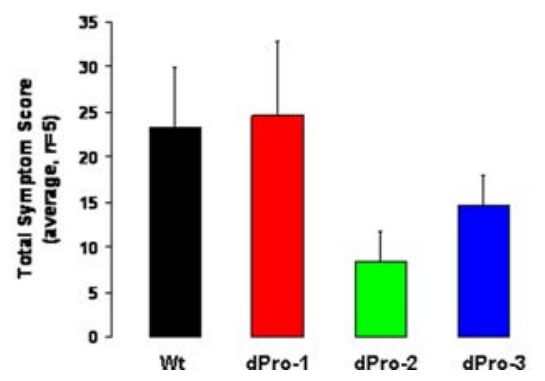

Fig. 3 Resistance analysis of transgenic and wild-type cassava plants to ACMV-NOg infection with $200 \mathrm{ng}$ viral DNA load per plant in the growth chamber. (a) Transgenic cassava lines dPro- 2 and dPro-3 were displayed attenuated disease symptoms on the 12 new emerged leaves post-inoculation, whereas non-transformed plants (Wt) and dPro-1 showed typical disease symptom development after virus inoculation. The bottom panel illustrates representative leaves showing different degrees of symptoms for the evaluation of symptom severity scores $(0=$ no symptoms, $4=$ severe symptoms). (b) Lines dPro- 2 and dPro-3 had reduced total symptom scores compared to Wt and dPro-1 profiles and newly emerging leaves were without visual symptoms (Fig. 4), in contrast to wild-type and dPro-1 plants. Because of the variability (oscillation) in the symptom appearance after ACMV infection (a shift of one leaf for the severity score generates a big standard variation), statistical analysis is difficult to perform. Therefore, we conducted another two independent experiments and both showed consistent results as we presented here. Our results suggest that transgenic cassava expressing siRNAs have enhanced phenotype recovery from ACMV infection.

Analysis of the virus titres in leaves at positions 4 postinoculation from phenotype recovery plants revealed that they are indeed reduced in the transgenic lines showing the recovery phenotype (Fig. 5). Virus titres in leaf 4 of line dPro-2 are at least reduced 4-fold if compared to the nontransformed line and about 6-fold in dPro-3. The ssDNA, which has been present in leaf 4 of wild-type, was still not detectable in leaf 4 of lines dPro- 2 and 3.

dsRNA transgene- and virus-derived short RNAs in cassava

Since the transgenic cassava plants are expressing an inverted repeat sequence of the ACMV CR-containing
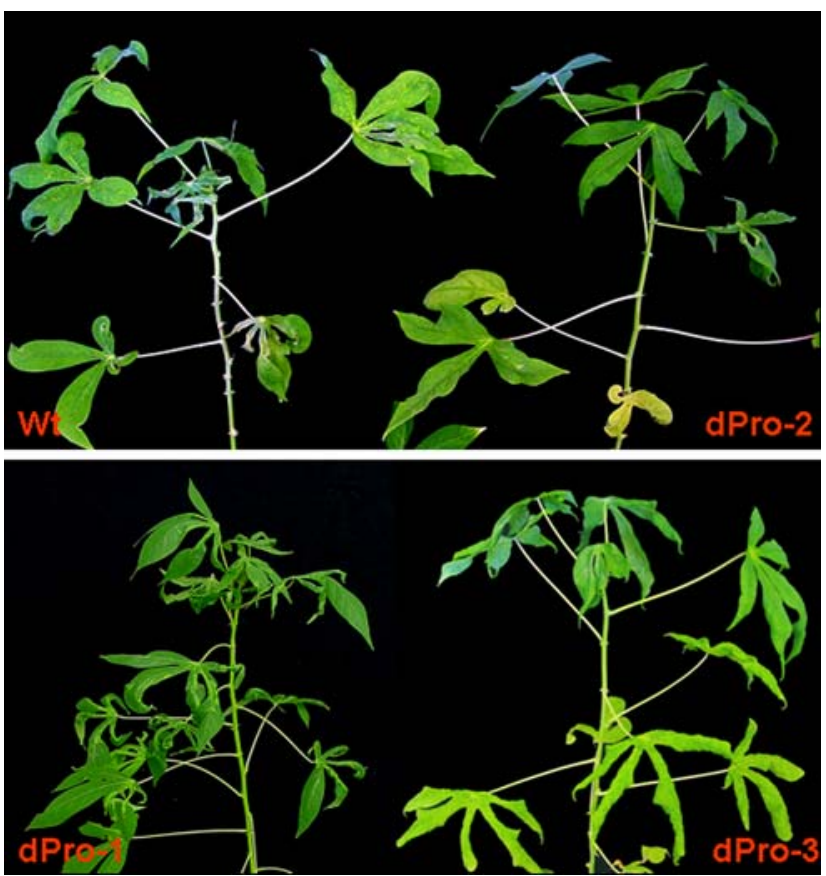

Fig. 4 Transgenic dPro-2 and dPro-3 cassava plants showed enhanced plant recovery from African cassava mosaic virus infection compared to wild-type (Wt) and dPro-1 plants under $200 \mathrm{ng}$ ACMVNOg DNA infection pressure. New leaves from dPro-2 and dPro-3 had reduced disease symptoms and Wt and dPro- 1 displayed typical disease symptoms, such as severe mosaic, distortion, size reduction etc 


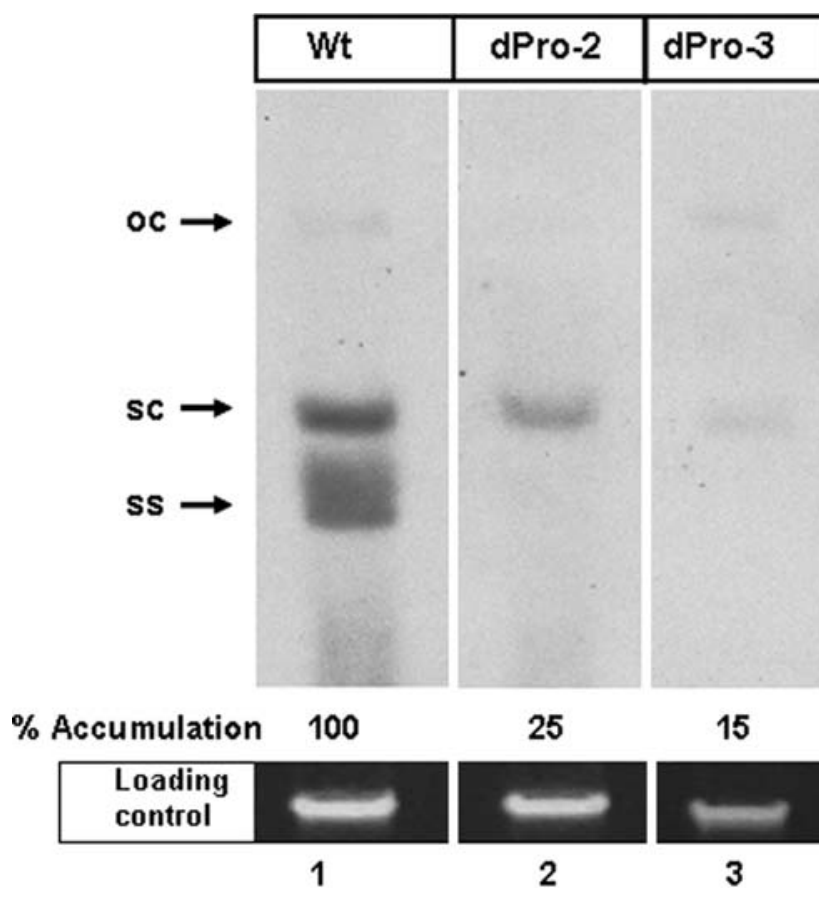

Fig. 5 Southern blot analysis of ACMV replication in wild-type and transgenic plants showing reduced viral DNA accumulation in resistant transgenic lines dPro-2 and dPro-3. Total DNA extracted from the 4th leaves of three infected plants per line was loaded for gel electrophoresis for DNA blotting. Viral DNA was detected using a DIG-labeled $A V 1$ probe. Viral single-stranded (ss), supercoiled (sc) and open-circular (oc) DNA forms are indicated

promoter, we expected that this product would fold into dsRNA and will consequently be processed into siRNAs by endogenous dicer-like enzymes. 21-24 nt siRNAs cognate to the CR region in sense and anti-sense were in fact detected in the transgenic lines dPro-2 and 3, while they were missing as expected in wild-type and also in the apparently inactive line dPro-1 (Fig. 6). Upon infection, siRNAs appeared in all lines and were also generated from the coding region of ACMV, such as $A C l$ and $B C l$. All siRNAs observed in infected wild-type and dPro- 1 are obviously derived from the infection, and the same is true for the siRNAs cognate to the ACMV coding region in transgenic lines dPro- 2 and 3 . The siRNAs cognate to the $\mathrm{CR}$ region in infected dPro- 2 and 3 are probably a mixture of those derived from the transgene and from the virus genomes. Thus, the enhanced recovery phenotype described above for the two transgenic lines dPro-2 and dPro-3 correlates positively with the accumulation of the transgene-derived siRNAs prior to infection. Overall, the pattern of transgene- and virus-derived siRNAs was similar, indicating they are produced by similar small RNA processing machinery. 21-nt siRNAs have been implicated in mRNA degradation, while 24-nt siRNAs in DNA methylation. Both of these species are produced from the transgene and the virus and could target viral mRNA and
DNA, respectively. Interestingly, 21- and 22-nt siRNAs were also detected for the coding regions of $A C l$ and $B C 1$, which are more abundant than the ACMV DNA-A bidirectional promoter-derived same classes of small RNAs. Virus-derived siRNAs are probably produced from illegitimate read-through transcripts as had been observed for another geminivirus (Shivaprasad et al. 2005).

Taken together, our results suggest that transgene-derived siRNAs could play a role in the observed recovery phenotype in transgenic cassava. It is consistent with previous observations in ACMV-infected cassava and Nicotiana in which recovery phenotype correlates with increased level of virus-derived siRNAs (Chellappan et al. 2004b).

\section{Discussion}

Our data showed that resistance to ACMV in the transgenic cassava is strongly associated with the expression of siRNAs prior to infection (Fig. 6). Two of our three transgenic lines, dPro-2 and dPro-3, were virus tolerant and in these two, viral DNA-A bidirectional promoterspecific siRNAs were present already prior to ACMV infection. After ACMV inoculation, all of the plants, including dPro- 1 and wild-type produced siRNAs with a profile similar to the transgene-derived siRNAs when using the same ACMV DNA-A promoter probes. siRNAs homologous to virus coding regions not present in the transgene, i.e. $A C l$ and $B C l$, also appeared after infection. They could have been derived from long dsRNA precursors produced upon read-through transcription (Shivaprasad et al. 2005) and/or by the action of RNAdependent RNA polymerases (RdRPs) on viral mRNAs (Xie et al. 2001). Blevins et al. (2006) showed that in Arabidopsis thaliana three size classes of siRNAs can be produced upon geminivirus infections and that these size classes can be assigned to specific dicers, 21-nt siRNAs to DCL4, 22-nt siRNAs to DCL2 and 24-nt siRNAs to DCL3. Our results show that all three size classes are produced in cassava from ACMV and ACMV-derived transgenic dsRNA sequences. The different size classes could be derived from dsRNAs of different origin, e.g. produced by read through transcription or RdRP, or from dsRNA present in different compartments (nucleus, cytoplasm). DCL3 producing the 24-nt siRNAs is thought to act mainly in the nucleus and this might explain, why 24-nt siRNAs are common in DNA virus infections (Blevins et al. 2006; Moissiard and Voinnet 2006), where viral RNA is produced in the nucleus, and rarely seen in RNA virus infections (Deleris et al. 2006; Blevins et al. 2006), where viral RNA is produced in the cytoplasm.

Virus-derived siRNAs have been associated earlier with geminivirus resistance in plants and an oscillation of viral 


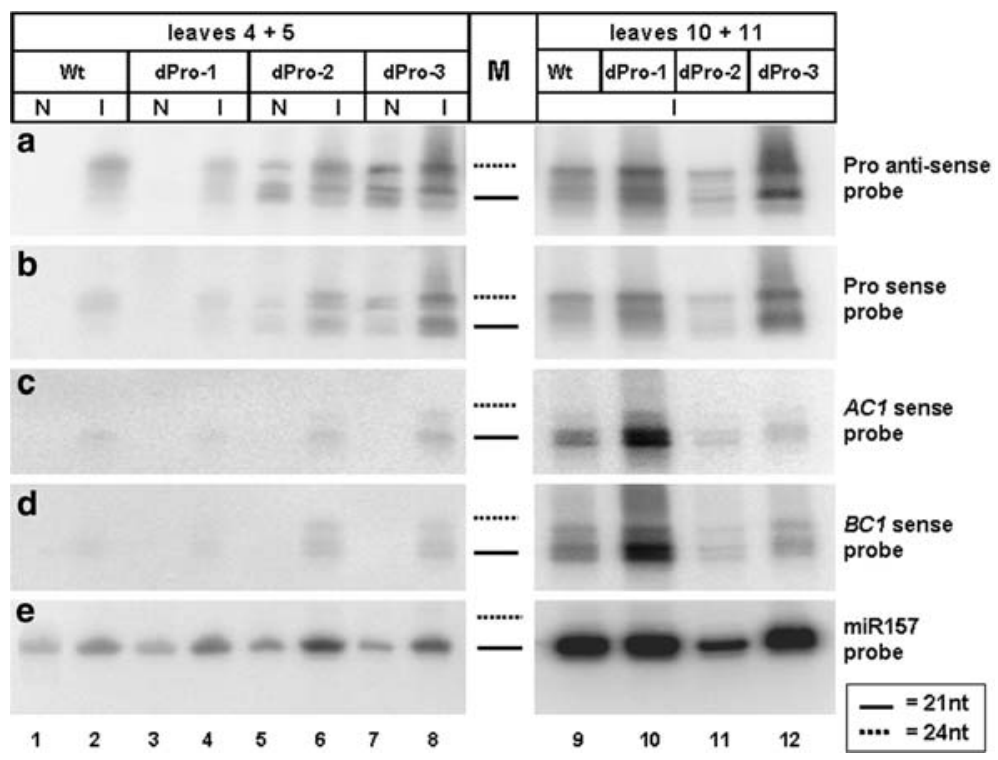

Fig. 6 RNA gel blot analysis of viral derived specific siRNAs in two leaf groups $(4+5 ; 10+11)$ of transgenic and wild-type $(\mathrm{Wt})$ plants using different probes. Without ACMV-NOg infection (N), siRNAs in sizes of 20-24 nt in length were detected using both anti-sense and sense probes from resistance lines dPro- 2 and dPro-3 (a, b; lanes 5 and 7) whereas no siRNA was detected in Wt and dPro-1 line (a, b; lanes 1 and 3). After infection (I), same sizes of siRNAs were detected in all infected plants (a, b; lanes 2, 4, 6, 8, 9-12). siRNAs profiles of ACMV-NOg infected $\mathrm{Wt}$ and transgenics were also detected using $A C 1$ and $B C 1$ probes. Small RNA miR157 was used as internal control

possible that only a few specific short RNAs are involved in RNA-directed DNA methylation. Methylation could then expand from the initially methylated islands to flanking regions, as proposed by Fojtova et al. (2003) and Van Houdt et al. (2003). In our transgenic cassava lines, the ACMV ssDNA is barely detectable while the dsDNA is still relatively abundant, but less than in wild-type cassava (Figs. 2, 5). The virus appears to be impeded for production of ssDNA via rolling circle replication. The viral promoter and CR might undergo siRNA-directed DNA methylation and histone modifications that reduce both the transcriptional activity of the promoter and/or impair the recruitment of DNA polymerase necessary for replication via altered Rep-binding site properties. Therefore, the reduced replication in the transgenic cassava leaves could be a direct consequence of an altered level of Rep transcription driven by the viral promoter. Further investigation of the relation between viral $\mathrm{CR}$ methylation status and transcription level of $A C l$ or $A V I$ is required in these transgenic lines.

It has been previously reported that virus titres do not necessarily correlate with symptom development (Cecchini et al. 1998; Chellappan et al. 2005; Lee et al. 1996). DNA forms and their ratios might better correlate with symptom development in infected plants. Besides a reduced viral titre in the transgenic lines, the ratio between viral dsDNA and ssDNA also differs between the transgenic lines and the wild-type. In both the leaf disk assay and plant methylation modification of the promoter region. It is also 
experiments, virus always produces a higher ratio of ssDNA/dsDNA in the wild-type than in the transgenic lines dPro-2 and dPro-3 (Figs. 2, 5).

Although our approach proved the possibility for developing cassava resistant to ACMV infection through siRNAs derived from non-coding sequences, the big disadvantage of this strategy is the low transformation efficiency. The regeneration frequency in this experiment is much lower than with other transformation events in our laboratory performed under a similar selection regime, in which a regeneration frequency of more than $60 \%$ could be achieved. To date, we have also produced dozens of ACMV-resistant transgenic cassava plants, which express siRNAs derived from ACMV coding sequences (unpublished data) and their efficiencies of cassava transformation were much higher. The low plant regeneration frequency can be due to several factors such as plant material quality and somaclonal variation during the tissue culture process. It also cannot be excluded that some siRNAs derived from the transgene might affect the development from somatic embryos to plant. It is also possible that the CR-containing transgene and its transcripts might sequester host replicase and/or replication factors that required for plant development.

In conclusion, our study demonstrates that resistance to geminiviruses in plants can be achieved via TGS and/or PTGS by expressing siRNA derived from non-coding viral sequences. As the common region of ACMV does not share a high degree of sequence homology $(<50 \%)$ with other cassava geminiviruses, e.g. EACMV and SACMV, the resistance is expected to be strain specific. Further investigation is required to check the robustness of the resistance to other cassava geminiviruses and performance of these transgenic cassava plants in the CMD epidemic field in Africa.

Acknowledgements We thank Dr. John Stanley (John Innes Centre) for the ACMV clones, Dr. Johannes Fütterer (ETH Zurich) for helpful discussions. This work was supported by grants from the Swiss Centre for International Agriculture (ZIL), the Eiselen-Foundation-Ulm, the Bill \& Melinda Gates Foundation through the Grand Challenges in Global Health initiative and the Indo-Swiss Collaboration in Biotechnology.

\section{References}

Akbergenov R, Si-Ammour A, Blevins T, Amin I, Kutter C, Vanderschuren H, Zhang P, Gruissem W, Meins F Jr, Hohn T, Pooggin MM (2006) Molecular characterization of geminivirusderived small RNAs in different plant species. Nucleic Acids Res 34:462-471

Blevins T, Rajeswaran R, Shivaprasad PV, Beknazariants D, SiAmmour A, Park HS, Vazquez F, Robertson D, Meins F Jr, Hohn T, Pooggin MM (2006) Four plant dicers mediate viral small RNA biogenesis and DNA virus induced silencing. Nucleic Acids Res 34:6233-6246
Cecchini E, Al-Kaff NS, Bannister A, Giannakou ME, McCallum DG, Maule AJ, Milner JJ, Covey SN (1998) Pathogenic interactions between variants of cauliflower mosaic virus and Arabidopsis thaliana. J Exp Bot 49:731-737

Chellappan P, Masona MV, Vanitharani R, Taylor NJ, Fauquet CM (2004a) Broad spectrum resistance to ssDNA viruses associated with transgene-induced gene silencing in Cassava. Plant Mol Biol 56:601-611

Chellappan P, Vanitharani R, Fauquet CM (2004b) Short interfering RNA accumulation correlates with host recovery in DNA virusinfected hosts, and gene silencing targets specific viral sequences. J Virol 78:7465-7477

Chellappan P, Vanitharani R, Ogbe F, Fauquet CM (2005) Effect of temperature on geminivirus-induced RNA silencing in plants. Plant Physiol 138:1828-1841

Chen YK, Lohuis D, Goldbach R, Prins M (2004) High frequency induction of RNA-mediated resistance against cucumber mosaic virus using inverted repeat constructs. Mol Breed 14:215-226

Deleris A, Gallego-Bartolome J, Bao J, Kasschau KD, Carrington JC, Voinnet O (2006) Hierarchical action and inhibition of plant Dicer-like proteins in antiviral defense. Science 313:68-71

Fojtova M, Van Houdt H, Depicker A, Kovarik A (2003) Epigenetic switch from posttranscriptional to transcriptional silencing is correlated with promoter hypermethylation. Plant Physiol 133:1240-1250

Goodall GJ, Filipowicz W (1989) The AU-rich sequences present in the introns of plant nuclear pre-mRNAs are required for splicing. Cell 58:473-483

Jørgensen K, Bak S, Busk PK, Sørensen C, Olsen CE, Puonti-Kaerlas J, Møller BL (2005) Cassava plants with a depleted cyanogenic glucoside content in leaves and tubers. Distribution of cyanogenic glucosides, their site of synthesis and tansport, and blockage of the biosynthesis by RNA interference technology. Plant Physiol 139:363-374

Kalantidis K, Psaradakis S, Tabler M, Tsagris M (2002) The occurrence of CMV-specific short Rnas in transgenic tobacco expressing virus-derived double-stranded RNA is indicative of resistance to the virus. Mol Plant Microbe Interact 15: $826-833$

Lee JM, Hartman GL, Domier LL, Bent AF (1996) Identification and map location of TTR1, a single locus in Arabidopsis thaliana that confers tolerance to tobacco ringspot nepovirus. Mol Plant Microbe Interact 9:729-735

Legg JP, Thresh JM (2003) Cassava virus diseases in Africa. In: Proceedings of the first international conference on plant virology in Sub-Saharan Africa. 4-8 June, Ibadan, Nigeria, pp 517-552

Liu SJ, Bedford ID, Briddon RW, Markham PG (1997) Efficient whitefly transmission of African cassava mosaic geminivirus requires sequences from both genomic components. J Gen Virol 78:1791-1794

Mette MF, Aufsatz W, van der Winden J, Matzke MA, Matzke AJ (2000) Transcriptional silencing and promoter methylation triggered by double-stranded RNA. Embo J 19:5194-5201

Moissiard G, Voinnet O (2006) RNA silencing of host transcripts by cauliflower mosaic virus requires coordinated action of the four Arabidopsis Dicer-like proteins. Proc Natl Acad Sci U S A 103:19593-19598

Pandolfini T, Molesini B, Avesani L, Spena A, Polverari A (2003) Expression of self-complementary hairpin RNA under the control of the rolC promoter confers systemic disease resistance to plum pox virus without preventing local infection. BMC Biotechnol 3:7-15

Pooggin MM, Shivaprasad PV, Veluthambi K, Hohn T (2003) RNAi targeting of DNA virus in plants. Nat Biotechnol 21:131-132 
Sambrook J, Fritsch EF, Maniatis T (1989) Molecular cloning: a laboratory manual, 2nd edn. Cold Spring Harbor Laboratory Press, Cold Spring Harbor

Shivaprasad PV, Akbergenov R, Trinks D, Rajeswaran R, Veluthambi K, Hohn T, Pooggin MM (2005) Promoters, transcripts, and regulatory proteins of Mungbean yellow mosaic geminivirus. J Virol 79:8149-8163

Smith NA, Singh SP, Wang MB, Stoutjesdijk PA, Green AG, Waterhouse PM (2000) Gene expression-Total silencing by intron-spliced hairpin RNAs. Nature 407:319-320

Soni R, Murray JAH (1994) Isolation of intact DNA and RNA from plant tissues. Analyt Biochem 218:474-476

Van Houdt H, Bleys A, Depicker A (2003) RNA target sequences promote spreading of RNA silencing. Plant Physiol 131:245-253

Vaucheret H, Fagard M (2001) Transcriptional gene silencing in plants: targets, inducers and regulators. Trends Genet 17:29-35

Wang MB, Abbott DC, Waterhouse PM (2000) A single copy of a virus-derived transgene encoding hairpin RNA gives immunity to barley yellow dwarf virus. Mol Plant Pathol 1:347-356
Waterhouse PM, Fusaro AF (2006) Viruses face a double defense by plant small RNAs. Science 313:54-55

Xie Z, Fan B, Chen C, Chen Z (2001) An important role of an inducible RNA-dependent RNA polymerase in plant antiviral defense. Proc Natl Acad Sci USA 98:6516-6521

Zhang P, Gruissem W (2003) Efficient replication of cloned African cassava mosaic virus in cassava leaf disks. Virus Res 92:47-54

Zhang P, Potrykus I, Puonti-Kaerlas J (2000a) Efficient production of transgenic cassava using negative and positive selection. Transgenic Res 9:405-415

Zhang P, Legris G, Coulin P, Puonti-Kaerlas J (2000b) Production of stably transformed cassava plants via particle bombardment. Plant Cell Rep 19:939-945

Zhang P, Vanderschuren H, Futterer J, Gruissem W (2005) Resistance to cassava mosaic disease in transgenic cassava expressing antisense RNAs targeting virus replication genes. Plant Biotechnol J 3:385-397 